\title{
Identificação Não-intrusiva de Cargas Similares em Smart Grid Usando Rede Neural Convolucional
}

\author{
Victor Pereira Firmes* Wanderley Cardoso Celeste** \\ Leonardo José Silvestre ${ }^{* * *}$ \\ * Programa de Pós-Graduação em Energia, Universidade Federal do \\ Espírito Santo, ES, (e-mail: victormecatronic@gmail.com). \\ ** Programa de Pós-Graduação em Energia, Universidade Federal do \\ Espírito Santo,ES, (e-mail: cawander@gmail.com) \\ *** Programa de Pós-Graduação em Energia, Universidade Federal do \\ Espírito Santo,ES, (e-mail: leosilvestre@gmail.com)
}

\begin{abstract}
This work deals with the problem of identifying technically identical equipments, defined here as highly similar equipments, monitored from a point of common coupling (PCC). An experimental approach is given here, where four highly similar computers are used, and none or even all computers can be in simultaneous operation. Each possible configuration of computers in simultaneous operation is defined here as a load. Samples of the electrical power demand for each load are acquired. A process of automatic extraction of load features is performed using convolutional neural networks $(\mathrm{CNN})$. These load features form the so-called load signature, which is the necessary and fundamental information for the training of the identification system, which proved to be very efficient when achieving a success rate of $98.96 \%$, i. e., considerably higher than those achieved by systems trained from manually obtained signatures.

Resumo: Este trabalho trata do problema de identificação de equipamentos monitorados a partir de um ponto comum de acoplamento, sendo tais equipamentos tecnicamente idênticos sob o ponto de vista elétrico, definidos aqui como equipamentos altamente similares. O problema é tratado neste artigo de forma experimental, onde são usados quatro computadores altamente similares, sendo que nenhum ou até todos os computadores podem estar em funcionamento simultâneo. Cada configuração possível de computadores em funcionamento simultâneo é aqui definida como carga. Amostras da potência elétrica demandada por cada carga são adquiridas. Um processo de extração automática de características das cargas é realizado utilizandose redes neurais convolucionais $(\mathrm{CNN})$. Tais características formam a chamada assinatura de carga, que é a informação necessária e fundamental para o treinamento do sistema de identificação, o qual se mostrou bastante eficiente ao se alcançar uma taxa de 98,96\% de precisão, apresentando, portanto, um desempenho muito superior a sistemas treinados a partir de assinaturas manualmente obtidas.
\end{abstract}

Keywords: Convolutional Neural Network; Smart Grid; Load Identification; Similar Loads. Palavras-chaves: Redes Neurais Convolucionais; Rede Elétrica Inteligente; Identificação de Cargas; Cargas Similares.

\section{INTRODUÇÃO}

A identificação de cargas elétricas tem se tornado importante pela crescente necessidade de um melhor gerenciamento do consumo de energia elétrica, assim como possibilitar a previsão da demanda, permitindo um melhor planejamento. Tal identificação é feita a partir das próprias características das cargas, conhecidas como assinaturas de carga (Lam et al., 2007).

Exitem duas abordagens para monitorar uma carga: monitoramento intrusivo (ILM) e o não intrusivo (NILM). No primeiro, é utilizado um medidor em cada carga e o monitoramento dos dispositivos é feito remotamente por um hardware complexo. Já no segundo caso, o monitoramento é feito através de um único dispositivo conectado à rede, o qual envia dados para uma central que é utilizada para reconhecer as cargas ativas (Rayudu et al., 2011).

O método não intrusivo apresenta vantagens frente ao intrusivo, tais como: custo baixo, facilidade na instalação e na manutenção, mesmo com a entrada de novos equipamentos na rede (Lan et al., 2017). Tais vantagens garantem a viabilidade de implantação do sistema de identificação, tornando o método NILM o mais investigado.

A Figura 1 apresenta um diagrama de blocos que constituem um sistema de identificação de cargas, onde o bloco Sistema de Aquisição representa um hardware capaz de obter amostras da potência (tensão e corrente) demandada pela carga, gerando assim uma massa de dados, a qual 


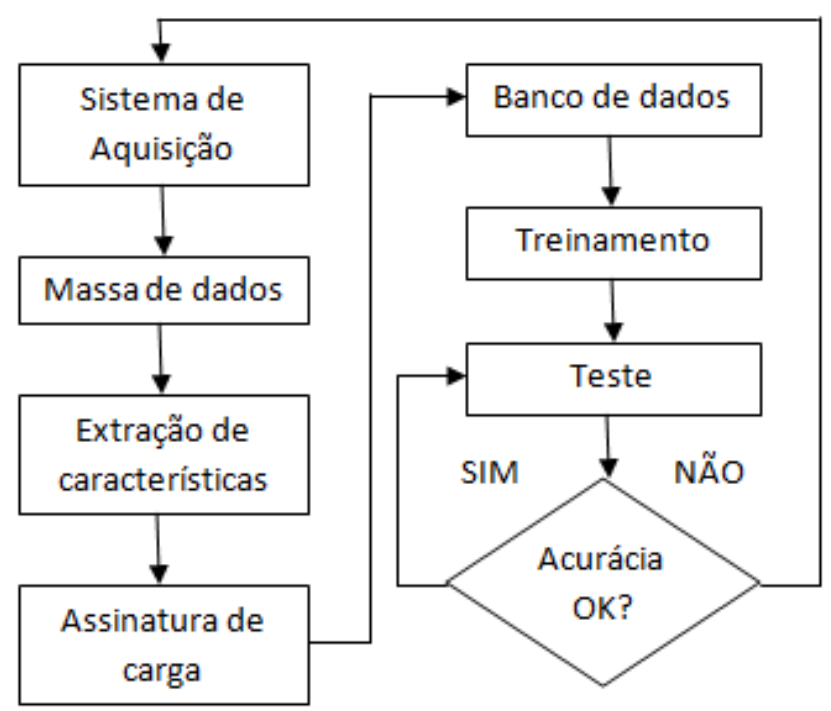

Figura 1. Diagrama de blocos do sistema de identificação de cargas.

alimenta um sistema de extração de características das cargas sob monitoramento.

O conjunto das características extraídas compõem a assinatura de cada carga monitorada, sendo tal assinatura a informação útil a qual é organizada em um banco de dados para treinamento de sistemas de identificação inteligente. Uma vez treinado, o sistema deve ser capaz de identificar com boa precisão o proprietário de cada nova assinatura de carga que for apresentada. Caso isso não seja possível, o processo deve se repetir. Assim é montado um banco de dados, o qual possibilita o treinamento de sistemas para identificação autônoma de cargas.

Os sistemas de identificação de cargas encontrados na literatura consideram cargas com baixo grau de similaridade. Logo, a taxa de sucesso de tais sistemas se degrada fortemente a medida que o grau de similaridade de tais cargas aumenta. O motivo de tal deficit de desempenho é o fato das características extraídas não servirem, de fato, como assinatura de carga em cenários envolvendo cargas altamente similares (Paixão, 2016), (Schneider, 2018). Logo, o sistema de identificação acaba sendo treinado para um caso muito específico de cargas com baixa similaridade.

Portanto, o problema de identificação está em se buscar as características que representem bem casos diversos, isto é, desde cargas com baixa similaridade até cargas altamente similares.

Dessa forma, este trabalho tem como objetivo propor um método de identificação de cargas não intrusivo, utilizando inteligência artificial para obter características escondidas que aparecem em cenários envolvendo cargas altamente similares. Assim, usando os dados elétricos da carga, treinar e testar o desempenho na identificação.

Nesse sentido, este artigo segue na Seção 2 com uma breve revisão da literatura, contextualizando o problema de identificação de cargas altamente similares através do método NILM, cuja metodologia e desenvolvimento são tratados na Seção 3. A Seção 4 traz os resultados experimentais alcançados e discussões sobre tais resultados, enquanto que na Seção 5 é apresentada a conclusão deste trabalho.

\section{REVISÃO DE LITERATURA}

A temática da identificação de cargas através do monitoramento não-intrusivo iniciou-se nos EUA na década de 90, objetivando a coleta de dados do uso final da eletricidade em residências. A potência ativa e a potência reativa foram utilizadas como características, usando uma frequência de amostragem de $1 \mathrm{~Hz}$ através de um único medidor de potência instalado. As cargas foram divididas em três classes: on-off, máquina de estado finito e continuamente variáveis. Na identificação, utilizou-se um algoritmo que era capaz de identificar cargas através da assinatura extraída previamente, disponíveis em um banco de dados. Apesar de o método conseguir identificar as cargas do tipo onoff e máquina de estado finito a uma baixa frequência de amostragem, o algoritmo não conseguia lidar com cargas mais complexas (continuamente variáveis) (Hart, 1992).

É destacado em Gursoy and Niebur (2009) que, devido ao aumento no número de equipamentos elétricos de potência e outras fontes harmônicas, a identificação das cargas harmônicas é um problema nos sistemas de transmissão e distribuição de energia elétrica. Eles pesquisaram sobre a técnica de processamento de sinais através da estatística para identificação das cargas. Nessa técnica, é possível estimar valores de tensão harmônica sem conhecer o sistema.

Em Gonzalez et al. (2012), a identificação de cargas foi realizada através dos sinais que se propagam no painel de medição de eletricidade de um edifício, utilizando a detecção das transições on-off da potência ativa, o nível de potência, a hora de acionamento e desligamento, o ciclo de serviço e o consumo de energia. O algoritmo construído foi capaz de identificar cargas com acurácia de até $90 \%$, porém somente para cargas com transições on-off que possuem níveis de potência de ativação e desativação iguais. Para cargas com variações contínuas de potência, nas quais há uma coincidência entre a ligação e o desligamento, o algoritmo não obteve sucesso em identificar a carga.

Já He et al. (2012) mostra uma abordagem para aplicação em edifícios inteligentes. No modelo, é utilizada uma estrutura baseada em um conhecimento prévio da carga, incluindo a identificação do circuito da fonte de alimentação front-end, princípio de operação elétrica e a utilização do cliente. Assim, é proposta uma taxonomia através da eletrônica de potência para garantir que represente as cargas elétricas reais. Foram realizados diversos testes em um protótipo de laboratório, apesar do pouco investimento e da quantidade de dados limitados, o método conseguiu reconhecer o uso de 16 equipamentos não-similares com acurácia de $98 \%$, utilizando a técnica da distância euclidiana e uma taxa de amostragem de $60 \mathrm{~Hz}$.

Em Hassan et al. (2014) foram avaliadas as assinaturas de cargas através das formas de onda de tensão e corrente (VI), potência ativa, reativa e distorção das harmônicas para classificar cargas de aparelhos residenciais. Para a classificação, eles utilizaram os seguintes algoritmos: rede neural e máquina de vetor de suporte (SVM). Através de um banco de dados abertos, conseguiram resultados acima de 98\% utilizando o estímulo adaptativo. Eles concluíram que 
o conjunto de vários algoritmos de classificação oferecem precisão, robustez e confiabilidade. Destacaram também a complexidade em identificar cargas similares.

É implementado em Belley et al. (2014) um sistema de identificação inteligente em uma residência composto por 16 equipamentos. Foi destacado que apesar da crescente demanda na utilização do monitoramento não-intrusivo de cargas e o constante lançamento de medidores inteligentes para a extração das assinaturas de cargas, tal hardware ainda apresentava um custo muito elevado e pouca acessibilidade. Além disso, as aplicações para esses sensores são apenas para redução do custo de consumo e economia de energia. No cenário com cargas similares, o sistema não foi capaz de reconhecê-las. Assim os 16 equipamentos foram conectados em fases diferentes. Eles atingiram uma acurácia de 98,3\% em eventos de liga e desliga das cargas não-similares.

Em Ahmadi and Martı (2015), as formas de onda de tensão e corrente são processadas e compiladas em uma biblioteca. Essa biblioteca contém medições projetadas no espaço de Eigenloads, que são auto vetores que descrevem o espaço das assinaturas de cargas. Dessa maneira, cada carga tem sua assinatura. Logo, cada medição é transformada em uma "foto" e um algoritmo é aplicado ao "conjunto de fotos". É gerada uma lista de todos os dispositivos ligados que pode ser acessada a qualquer momento. Assim, esse método pode ser implantado em medidores inteligentes, possibilitando um processamento distribuído, permitindo assim que sejam realizados cálculos mais simples em cada medidor sem a necessidade de ter bancos de dados e um processador central poderoso. Apesar do sistema ter alcançado uma taxa de acerto de $100 \%$, ele não foi capaz de distinguir equipamentos resistivos.

Em Kelly and Knottenbelt (2015) foram adaptadas três arquiteturas de rede neural, isto é, a rede neural recorrente (LSTM), a rede autoencoder e a rede que regula o tempo de início, o tempo final e a demanda média de energia de cada ativação do dispositivo. Todas as três redes atingiram valores de acurácia melhores (76-100\%) do que a otimização combinatória ou modelo fatorial oculto de Markov.

Foi apontado em Singh et al. (2015) a dificuldade em identificar cargas similares no uso doméstico. Eles utilizaram coeficientes de wavelet, que são dados de entrada para um classificador de cargas. As cargas utilizadas foram CPU, monitor de LCD e notebook, enquanto que as classes foram a combinação das 3 cargas, num total de 7 classes. Foram considerados diversos algoritmos de classificação, dentre eles, o melhor desempenho foi a máquina de vetor de suporte (SVM) para cargas similares com uma acurácia de $100 \%$.

No estudo de Paixão (2016) foi proposto um sistema de classificação inteligente de cargas elétricas similares usando amostras de sinais de tensão e de corrente. Para isso, montou-se uma plataforma experimental com 4 equipamentos similares, os quais foram colocados em funcionamento simultâneo usando todas as combinações possíveis, o que resultou em 16 configurações. Dessas configurações foram extraídos 150 casos com 14 características elétricas manualmente obtidas. O autor, por fim, usou o conjunto de casos disponível para treinar e testar cinco classificadores, no qual obteve-se uma taxa de acerto de até $72,5 \%$. Vale ressaltar que o autor introduziu experimentos para tratar o problema de identificação de cargas similares.

No estudo de Sadeghianpourhamami et al. (2017), propõemse uma eliminação de características que não são importantes para a identificação de cargas e criam um conjunto de dados que pode ser usado para comparação. O algorítimo proposto alcançou uma acurácia de $100 \%$ para cargas distintas de uma residência. A carga que teve a menor taxa de acerto foi o aquecedor, pois ele é confundido com outros equipamentos resistivos que possuem potências parecidas. O problema da similaridade também não foi abordado, ressaltando a lacuna que existe neste tipo de identificação.

Já Schneider (2018) usou a entropia de Shannon e Renyi otimizada como características extraídas do mesmo banco de amostras usado por Paixão (2016). Inclusive, os mesmos classificadores foram treinados e testados a partir de tais características, obtendo uma taxa de acerto de $77,31 \%$, o que representou uma melhoria do sistema de identificação envolvendo o cenário de cargas com graus de similaridade diversos, como proposto inicialmente em Paixão (2016). Apesar disso, o erro de identificação para um uso prático ainda era considerado alto, requerendo mais esforços de pesquisa na busca por características que, de fato, atuem como assinaturas de carga.

Observa-se através da revisão de literatura que há uma forte diminuição das taxas de acerto a partir do momento em que se tenta identificar cargas altamente similares usando características tradicionalmente utilizadas. Ainda é possível observar que, a partir do momento em que se incorpora técnicas computacionais para extração e seleção de características, tais taxas aumentam, mostrando, portanto, o possível caminho para se lidar com esse complexo problema.

\section{METODOLOGIA}

Para resolver o problema de identificação autônoma de cargas, usa-se uma metodologia que é detalhada a seguir, onde na Subseção 3.1 apresenta-se o sistema de aquisição de amostras de sinais de potência demandada por um conjunto definido de cargas, enquanto que na Subseção 3.2 apresenta-se a ferramenta de extração autônoma de características e de identificação de cargas.

\subsection{Sistema de Aquisição de Dados}

Para a criação do banco de dados com as 16 configurações possíveis de funcionamento (Tabela 1) foi montada uma bancada experimental (Figura 2) composta por microcomputador rodando a interface gráfica do sistema de aquisição de dados, o módulo sensorial contendo o sensor de corrente e de tensão (modelo LA55-P e LV20-P), o ponto de acoplamento comum (PCC) com capacidade de conectar até 4 equipamentos e o módulo de aquisição de dados NI-cDAQ-9174 com o cartão NI-9220, ambos da National Instruments.

Como equipamentos altamente similares acoplados no PCC, foram utilizados 4 microcomputadores Dell Optiplex 960 Core 2 Duo $3 \mathrm{GHz}$, 4GB RAM, HD de 150GB e Potência de 255 W. Entre o PCC e o módulo de Aquisição 


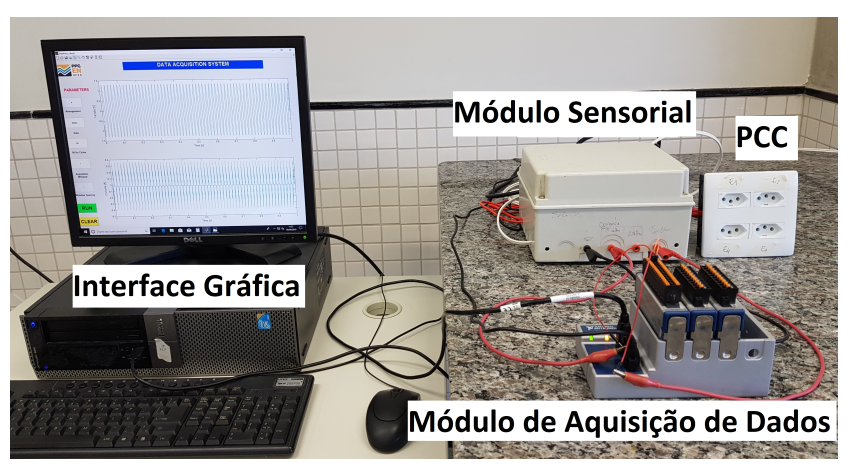

Figura 2. Bancada Experimental.

está conectado o módulo Sensorial, permitindo assim a observação e a captura de amostras da potência (tensão e corrente) demandada pela carga, isto é, fluindo pelo PCC.

Tabela 1. Configurações das cargas.

\begin{tabular}{cccccc} 
Classes & C4 & C3 & C2 & C1 & Estado \\
\hline 0 & 0 & 0 & 0 & 0 & Todos desligados \\
1 & 0 & 0 & 0 & 1 & C1 ligado \\
2 & 0 & 0 & 1 & 0 & C2 ligado \\
3 & 0 & 0 & 1 & 1 & C1/C2 ligados \\
4 & 0 & 1 & 0 & 0 & C3 ligado \\
5 & 0 & 1 & 0 & 1 & C1/C3 ligados \\
6 & 0 & 1 & 1 & 0 & C2 $/ \mathrm{C} 3$ ligados \\
7 & 0 & 1 & 1 & 1 & C1/C2/C3 ligados \\
8 & 1 & 0 & 0 & 0 & C4 ligado \\
9 & 1 & 0 & 0 & 1 & C1/C4 ligados \\
10 & 1 & 0 & 1 & 0 & C2 $/ \mathrm{C} 4$ ligados \\
11 & 1 & 0 & 1 & 1 & C1/C2/C4 ligados \\
12 & 1 & 1 & 0 & 0 & C3/C4 ligados \\
13 & 1 & 1 & 0 & 1 & C1/C3/C4 ligados \\
14 & 1 & 1 & 1 & 0 & C2/C3/C4 ligados \\
15 & 1 & 1 & 1 & 1 & Todos ligados \\
\hline
\end{tabular}

A aquisição de dados se divide em 3 etapas, isto é configuração, aquisição e armazenamento. Na etapa de configuração é feito o ajuste de parâmetros do módulo de Aquisição via interface gráfica desenvolvida para facilitar tal tarefa, bem como exibir uma forma gráfica dos sinais amostrados. Os parâmetros de configuração são: o arranjo de carga ligada/desligada classes 0 a 15, conforme Tabela 1, a taxa de captura do amostrador em $\mathrm{Hz}$, a quantidade de ciclos de $60 \mathrm{~Hz}$, a quantidade de janelas de aquisição e o espaço temporal entre essas janelas. Cabe ressaltar que tal espaço temporal entre janelas de aquisição pode ser regular ou aleatório, a critério do operador.

No caso específico, para replicar situações reais, as amostras foram capturadas aleatoriamente dentro do período que compreende as janelas de aquisição, tendo em vista que, tais situações, as condições de funcionamento de equipamentos mudam de forma não determinística, o que reflete em uma variação aleatória da potência demandada.

Para garantir que o conjunto de amostras a ser adquirido equivalha a um número inteiro de ciclos de $60 \mathrm{~Hz}$, é importante ajustar o módulo de aquisição para uma frequência de amostragem que seja múltipla de $60 \mathrm{~Hz}$. No caso em específico, usou-se a frequência de $99960 \mathrm{~Hz}$ (a maior frequência do módulo NI-9220 é $100 \mathrm{kHz}$ ), de modo que cada ciclo de $60 \mathrm{~Hz}$ é representado por 1666 amostras.
Para garantir uma grande quantidade de amostras para a aplicação da ferramenta CNN (vide Subseção 3.2), foram adquiridas 10 janelas com largura 60 ciclos cada (ou 1 s), com espaçamento aleatório entre as janelas, como já mencionado.

Portanto, para cada uma das 16 configurações apresentadas na Tabela 1, foram adquiridas 999600 amostras, o que equivale a 600 ciclos de $60 \mathrm{~Hz}$, ou 600 casos, totalizando 9600 casos contendo 1666 amostras cada. Embora o módulo de Aquisição capture tensão e corrente demandadas pela carga, optou-se neste trabalho em usar somente as amostras de corrente, tendo em vista que a para as cargas usadas a tensão se mantém constante.

Por fim, na etapa de armazenamento, todos os 9600 casos foram armazenados no Google Colab. Essa ferramenta permite o uso de uma máquina virtual com GPU modelo NVIDIA Tesla K80 12 GB de memória RAM gratuita, ambiente de programação em Python 3 e bibliotecas de deep learning (que contém a ferramenta utilizada para treinamento e classificação autônoma apresentada na Subseção 3.2) como o Keras e o TensorFlow. Cabe mencionar que é fundamental o uso de GPU nas CNNs (Subseção 3.2) por causa da grande quantidade de parâmetros a serem ajustados.

\subsection{Caracterização e Classificação Autônoma}

As redes neurais convolucionais (Convolutional Neural Network - CNNs)) são redes com estruturas em convolução que usa a estratégia de compartilhamento de peso, reduzindo a complexidade do modelo da rede, consequentemente, a quantidade de memória utilizada. Nos últimos anos, as CNNs conseguiram solucionar diversos problemas de visão computacional, como classificação de imagens, detecção de objetos e alteração de imagens. Tudo isso foi possível devido à sua capacidade de selecionar características em diferentes níveis (Lan et al., 2017).

Diversos trabalhos vêm utilizando as CNNs para resolver problemas relativos à classificação. Em LeCun et al. (1998) os autores compararam vários métodos de reconhecimento de caracteres manuscritos com um padrão. As CNNs para formas bidimensionais (2D) superaram todos os outros métodos de identificação. Em Krizhevsky et al. (2012), Simonyan and Zisserman (2014), Szegedy et al. (2015) e He et al. (2016) é mostrado como as taxas de acerto foram melhorando a cada alteração feita na rede CNN (de $62,5 \%$ para $96,4 \%$ ) para o problema de classificação de imagens com três canais de cores.

Uma das vantagens da CNN é que ela usa dados brutos, sendo o pré-processamento a sua primeira etapa de funcionamento. No caso em específico, utilizou-se duas opções de normalização de dados como parte do pré-processamento da CNN, isto é, MinMaxScaler e StandardScaler. Na sequência, a CNN converte os valores das amostras de corrente de inteiro para binário. A Figura 3 apresenta a arquitetura generalizada da CNN adotada. Pode-se observar na figura que a rede neural desempenha duas tarefas, isto é, realiza a caracterização (extração automática das características) e realiza a classificação autônoma de novos casos apresentados. O treinamento da rede como um todo é feito usando-se $64 \%$ dos casos, sendo tal treinamento 


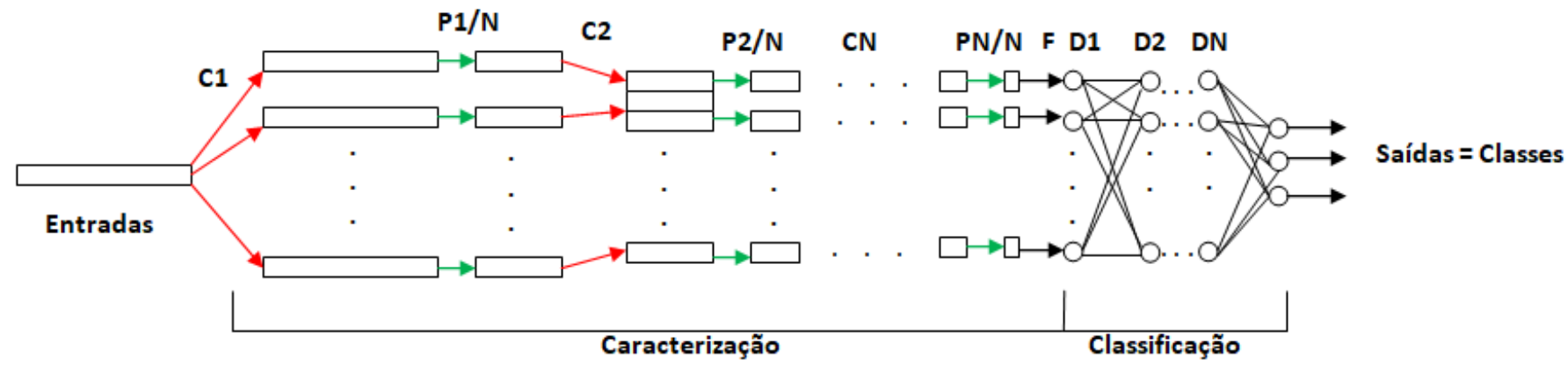

C: Camada convolucional com X filtros 1D de comprimento Y P: Camada Maxpooling com tamanho Z N: Camada de Normalização do Batch F: Camada Flatten D: Camada densa com W neurônios

Figura 3. Arquitetura generalizada da rede.

validado usando-se outros $16 \%$ dos casos. Os $20 \%$ dos casos restantes são usados para testar a rede treinada e validada.

As etapas de treinamento e validação foram repedidas a cada variação de parâmetros configuráveis da CNN, a saber: tamanho do batch, número de épocas, tipo de otimizador, quantidade de filtros e de neurônios. Cada rede treinada e validada foi testada, gerando assim uma matriz de confusão, na qual é destacada a acurácia de cada classe.

\section{RESULTADOS E DISCUSSÃO}

Foram treinadas e testadas 5 redes CNN no servidor da Google. Os principais parâmetros ajustados nas redes (Batch, Pré-Proc. e Otimiz.) e as principais métricas para avaliação de desempenho (Acur. e Parâm.) são mostrados na Tabela 2. Observa-se na tabela que a rede 5 foi a que apresentou a melhor acurácia, com 98,96\%, mostrando que é eficiente na predição de 11 classes com 100\% de acerto, conforme pode ser visto na matriz de confusão da Figura 4.

Tabela 2. Resultados de CNNs.

\begin{tabular}{cccccc} 
Rede & Batch & Pré-Proc. & Otimiz. & Acur. $(\%)$ & Parâm. \\
\hline 1 & 32 & MinMax & Adam & 92,45 & 148.652 \\
2 & 32 & Standard & SGD & 87,03 & 148.652 \\
3 & 32 & MinMax & SGD & 95,57 & 148.844 \\
4 & 15 & Standard & SGD & 98,85 & 2.296 .791 \\
5 & 32 & Standard & SGD & 98,96 & 2.968 .800 \\
\hline
\end{tabular}

Apesar da rede 5 ter apresentado a melhor acurácia, ela também apresentou um grande número de parâmetros, um total de 2.928.800. Isso se deve ao fato da arquitetura possuir mais filtros e mais camadas quando comparada as redes 1,2 e 3, cuja quantidade de parâmetros ficou em cerca de $20 \%$.

A Figura 4 apresenta a matriz de confusão da rede 5, detalhando seu desempenho, podendo-se destacar a alta eficiência da rede em se identificar com grande precisão praticamente todas as 16 classes do problema em questão. Destaca-se ainda as situações de confusão da rede, as quais ocorrem sempre quando há cargas altamente similares envolvidas. Por exemplo, classe 2 confundida com classe 1 (ambas correspondem a apenas 1 equipamento ligado, conforme Tabela 1) e classe 10 confundida com classe 9 (ambas correspondem a 2 equipamentos ligados, conforme Tabela 1).

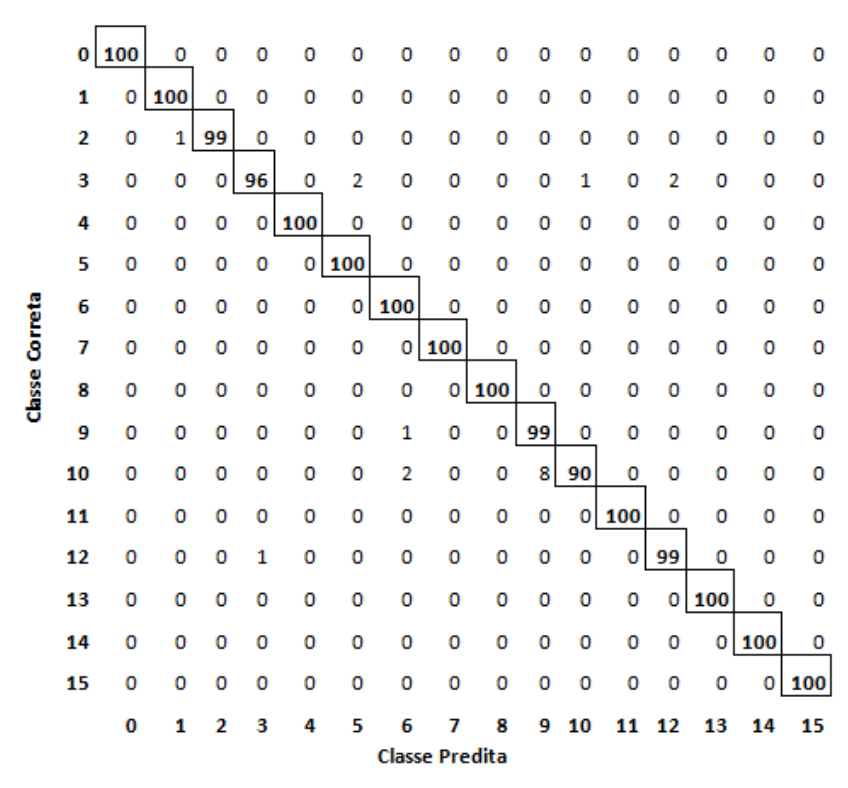

Figura 4. Matriz de confusão em percentual de acerto

Como já mencionado, a Figura 3 retrata uma arquitetura generalizada das CNNs implementadas. No caso específico da Rede 5, tomada como exemplo para uma análise mais aprofundada dos resultados, foram utilizadas 4 camadas de convolução, pooling e normalização do batch, 1 camada flatten e 4 camadas densas. As camadas de convolução apresentaram respectivamente 32, 64, 128 e 256 filtros de uma dimensão, com comprimento (kernel) igual a 4 , passo (stride) igual a 1 e função de ativação Rectified Linear Units (ReLU). As camadas de pooling foram do tipo Maxpooling com tamanho 3. O número de neurônios de cada camada densa foi de 512, 256, 128 e 16. As três primeiras foram ativadas pela função ReLU e a última com a função softmax, a qual determina a probabilidade de cada uma das 16 classes do problema.

$\mathrm{Na}$ rede 4, o batch foi reduzido para 15, e os filtros das camadas de convolução foram alterados para 50, 70, 100 e 200, com o mesmo comprimento e passo da rede 5 , enquanto que o número de neurônios das 4 camadas densas subsequentes foi de 500, 250, 175 e 16 respectivamente. Comparando os resultados da Tabela 2, é possível observar que houve uma redução na acurácia de $0,11 \%$ em função de uma redução de aproximadamente $23 \%$ do número de parâmetros da rede. 
A Figura 5 permite uma análise da acurácia da Rede 5 em função do número de épocas utilizadas em seu processo de treinamento e validação. Observa-se na figura que a partir de 500 épocas o sistema fica estável e não consegue melhorar sua acurácia. No cenário de maior acurácia, tal estabilização acontece após a época 384. Logo, o tempo de treinamento da rede em questão poderia ser reduzido em cerca de $62 \%$ sem prejuízo de desempenho.

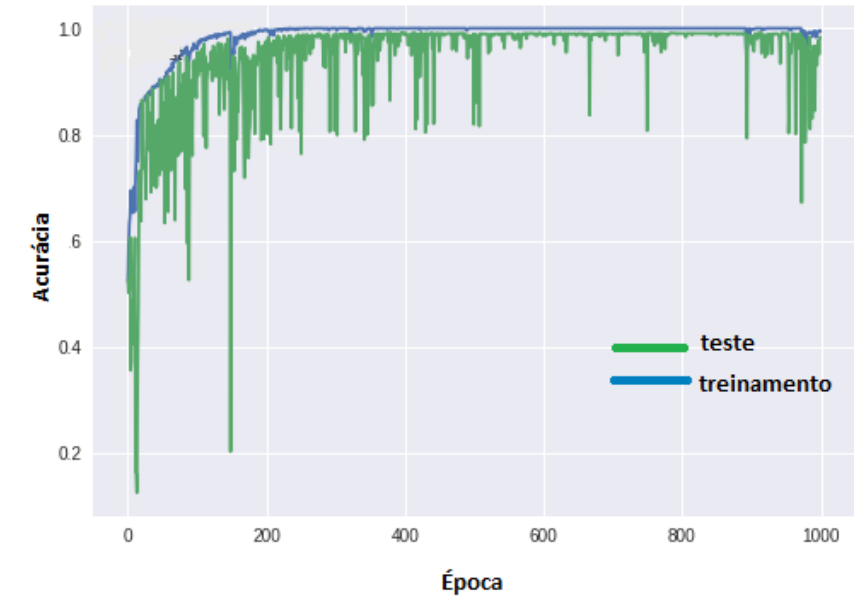

Figura 5. Evolução do treinamento e teste da rede 5.

\section{CONSIDERAÇÕES FINAIS}

Neste artigo foi proposta uma metodologia para a identificação de cargas elétricas similares em Smart Grid, usando um mecanismo de extração automática de características a partir do dado bruto, baseado em CNN. Através do ajuste dos parâmetros da CNN foi possível obter redes que proporcionam uma alta acurácia em cenário envolvendo, inclusive, cargas altamente similares.

\section{AGRADECIMENTOS}

Os autores deste artigo agradecem a CAPES pelo apoio financeiro (proc. 1779459/18).

\section{REFERÊNCIAS}

Ahmadi, H. and Martı, J.R. (2015). Load decomposition at smart meters level using eigenloads approach. IEEE Transactions on Power Systems, 30(6), 3425-3436.

Belley, C., Gaboury, S., Bouchard, B., and Bouzouane, A. (2014). An efficient and inexpensive method for activity recognition within a smart home based on load signatures of appliances. Pervasive and Mobile Computing, 12, 58-78.

Gonzalez, M., Debusschère, V., and Bacha, S. (2012). A load identification method for residential building applications. In Industrial Technology (ICIT), 2012 IEEE International Conference on, 84-88. IEEE.

Gursoy, E. and Niebur, D. (2009). Harmonic load identification using complex independent component analysis. IEEE Transactions on Power Delivery, 24(1), 285-292.

Hart, G.W. (1992). Nonintrusive appliance load monitoring. Proceedings of the IEEE, 80(12), 1870-1891.
Hassan, T., Javed, F., and Arshad, N. (2014). An empirical investigation of vi trajectory based load signatures for non-intrusive load monitoring. IEEE Trans. Smart Grid, 5(2), 870-878.

He, D., Du, L., Yang, Y., Harley, R., and Habetler, T. (2012). Front-end electronic circuit topology analysis for model-driven classification and monitoring of appliance loads in smart buildings. IEEE Transactions on Smart Grid, 3(4), 2286-2293.

He, K., Zhang, X., Ren, S., and Sun, J. (2016). Deep residual learning for image recognition. In Proceedings of the IEEE conference on computer vision and pattern recognition, 770-778.

Kelly, J. and Knottenbelt, W. (2015). Neural nilm: Deep neural networks applied to energy disaggregation. In Proceedings of the 2nd ACM International Conference on Embedded Systems for Energy-Efficient Built Environments, 55-64. ACM.

Krizhevsky, A., Sutskever, I., and Hinton, G.E. (2012). Imagenet classification with deep convolutional neural networks. In Advances in neural information processing systems, 1097-1105.

Lam, H.Y., Fung, G., and Lee, W. (2007). A novel method to construct taxonomy electrical appliances based on load signaturesof. IEEE Transactions on Consumer Electronics, 53(2).

Lan, Z., Yin, B., Wang, T., and Zuo, G. (2017). A nonintrusive load identification method based on convolution neural network. In Energy Internet and Energy System Integration (EI2), 2017 IEEE Conference on, 15. IEEE.

LeCun, Y., Bottou, L., Bengio, Y., and Haffner, P. (1998). Gradient-based learning applied to document recognition. Proceedings of the IEEE, 86(11), 2278-2324.

Paixão, A. (2016). Classificação Inteligente Aplicada ao Problema de Identificação de Cargas Elétricas "Idênticas". Master's thesis, Universidade Federal do Espírito Santo.

Rayudu, R., Tyler, C., and Witherden, M. (2011). Towards a tool for non-intrusive individual load measurement and identification. In Innovative Smart Grid Technologies Asia (ISGT), 2011 IEEE PES, 1-8. IEEE.

Sadeghianpourhamami, N., Ruyssinck, J., Deschrijver, D., Dhaene, T., and Develder, C. (2017). Comprehensive feature selection for appliance classification in nilm. Energy and Buildings, 151, 98-106.

Schneider, R. (2018). Identificação inteligente de cargas elétricas similares em Smart Grid. Master's thesis, Universidade Federal do Espírito Santo.

Simonyan, K. and Zisserman, A. (2014). Very deep convolutional networks for large-scale image recognition. arXiv preprint arXiv:1409.1556.

Singh, M., Kumar, S., Semwal, S., and Prasad, R. (2015). Residential load signature analysis for their segregation using wavelet-svm. In Power Electronics and Renewable Energy Systems, 863-871. Springer.

Szegedy, C., Liu, W., Jia, Y., Sermanet, P., Reed, S., Anguelov, D., Erhan, D., Vanhoucke, V., and Rabinovich, A. (2015). Going deeper with convolutions. In Proceedings of the IEEE conference on computer vision and pattern recognition, 1-9. 